\title{
$\gamma$-Cygni: the GeV to TeV morphology of an unique Sedov-phase SNR with MAGIC and Fermi-LAT
}

\author{
Marcel C. Strzys*, levgen Vovk, Christian Fruck \\ Max Planck Institute for Physics, Munich, Germany \\ E-mail: strzys@mpp.mpg.de, Ievgen.Vovk@mpp.mpg.de, \\ fruck@mpp.mpg . de
}

\section{Shu Masuda, Takayuki Saito}

Kyoto University, Kyoto, Japan

E-mail: masuda@cr.scphys.kyoto-u.ac.jp,

tysaitodcr.scphys.kyoto-u.ac.jp

\section{for the MAGIC Collaboration}

\begin{abstract}
The Gamma Cygni supernova remnant (G78.2+2.1) is a middle-aged SNR ( 7000 years old $)$ situated in the Cygnus region. The high-energy observations by VERITAS and Fermi-LAT revealed a complex, energy-dependent morphology of the SNR in the GeV-TeV band, different from that observed in X-rays. G78.2+2.1 also hosts the pulsar PSR J2021+4026, which is the only variable gamma-ray pulsar known to date. Using recent observations of the Gamma Cygni region with the MAGIC telescopes together with data from the Fermi-LAT, we present the structure of the emission from the northern and southern shell in the $\mathrm{GeV}$ to $\mathrm{TeV}$ energy range. We discuss the implication of the result of this morphological analysis in the context of our current understanding of supernova remnant models and view possible counterparts of the emission in a multi-wavelength context.
\end{abstract}

35th International Cosmic Ray Conference - ICRC2017

10-20 July, 2017

Bexco, Busan, Korea

* Speaker.

${ }^{\dagger}$ now at ICRR, The University of Tokyo 


\section{Introduction}

Recently the Fermi-Collaboration proved the long expected hypothesis that supernova remnants (SNRs) can accelerate cosmic rays up to TeV energies [1]. Those objects were however matured SNRs $\left(\approx 10^{4} \mathrm{yrs}\right)$ at the end of the Sedov phase, already interacting with the interstellar medium (ISM) and not showing coincidence between $\gamma$-ray emission and a bright radio shell any more. Younger Sedov SNRs do show $\gamma$-ray bright limb emission, but which spectra seem to be better explained by leptonic models [2]. The $\gamma$-Cygni SNR, being $\sim 7000$ yrs old and in the middle of the Sedov phase, may fill this gap and may allow to study the evolution of SNR over the Sedov phase and thus the transition of the bright SNR shell towards SNR/Molecular cloud interaction. This phase is connected with the deceleration of the shock and an increased escape of cosmic rays as the SNR interacts more with the ISM.

The supernova remnant G 78.2+2.1 is situated in the heart of the Cygnus region, close to the bright $\gamma$-Cygni star Sadr. Hence, it is also called the $\gamma$-Cygni supernova remnant. It is the remnant of a core-collapse supernova hosting the pulsar PSR J 2021+4026 that can be associated with the SNR [3], the only known $\gamma$-ray-variable pulsar [4]. The remnant shows a bright circular radio shell with a radius of $60^{\prime}$ [5]. The system lies at a distance of 1.5-2.6 kpc ([6],[7]) from the Earth. Assuming a distance of $1.7 \mathrm{kpc}$ the radial extend of the shell is $17 \mathrm{pc}$.

It was one of the first $\gamma$-ray sources discovered in 1979 by the COS-B satellite. Since then it has been studied by various instruments over the whole electromagnetic spectrum. The VERITAS telescopes found $\mathrm{TeV} \gamma$-ray emission in 2013 towards the northern part of the shell ([8]) that does not spatially coincide with the GeV emission seen by Fermi-LAT, which seems rather distributed all over the SNR shell ([9]). Fraija and Araya [10] found that the GeV emission seen by Fermi-LAT shows a harder spectrum at the VERITAS source than for the rest of the shell. Still the connection between the $\mathrm{GeV}$ shell and the $\mathrm{TeV}$ extended source remains unclear.

\section{MAGIC data taking and analysis}

The MAGIC (Major Atmospheric Gamma Imaging Cherenkov) telescopes are a system of two $17 \mathrm{~m}$ diameter large Imaging Air Cherenkov Telescopes (IACTs). The telescopes are located at the Roque de los Muchachos Observatory on the Canary island La Palma, Spain, at $2200 \mathrm{~m}$ altitude. The telescopes observe the short flashes of Cherenkov light induced by $\gamma$-ray air showers in stereoscopic mode. When operated in standard mode the telescopes cover the energy range from $50 \mathrm{GeV}$ to more than $50 \mathrm{TeV}$. The energy resolution is $16 \%$ and the angular resolution of the system is $<0^{\circ} .07$. The integral sensitivity in $50 \mathrm{~h}$ above $220 \mathrm{GeV}$ is $0.66 \%$ Crab. [11]

The data set presented here comprises $45 \mathrm{~h}$ of dead-time-corrected observation time after selection cuts taken between May and November 2015. The data were recorded under dark conditions between $10^{\circ}$ and $43^{\circ}$ Zenith distance. Data of reasonable quality with an atmospheric transmission, measured by a LIDAR system [12], of $>80 \%$ were included. In order to minimise the effect of the nearby bright star Sadr (2.2 mag) two wobble pointings were chosen around the VERITAS source position in the northern part of the shell. Still the star affects the recorded shower images raising the analysis threshold to $200 \mathrm{GeV}$. The point spread function (PSF) (for $\mathrm{E}>200 \mathrm{GeV}$ ) during the observational period is estimated using point-sources to be $\leq 0^{\circ} .065$ ( $\sigma$ extend of 2D Gaussian). 
The data reduction was performed using the standard MAGIC Analysis and Reconstruction Software (MARS). After the $\gamma /$ hadron separation carried out by a Random Forest classifier [13], the high level data are analysed using a newly developed spatial 2D likelihood analysis similar to what is used for Fermi-LAT data and based on [14]. It consists of a package computing the 2D instrument response functions such as the PSF and the exposure from Monte-Carlo simulations based on the pointing history of the telescope and obtaining the background model using all recorded events except for the ones arriving in a predefined source region. For this study the area of the radio source and a $0^{\circ} .5$ region towards the northern part of the shell was excluded from the background model. The models are constructed in camera coordinates and afterwards projected on the sky considering the rotation between the camera system and RA/Dec coordinates. This new analysis tool allows us for the first time to fit multiple source components to data obtained with an IACT and to extract their spectra simultaneously.

\section{Fermi-LAT data}

The data from the Fermi-LAT presented here are reduced using the Pass8 (P8R2_V6) analysis and the event selection cuts for the P8R2_SOURCE event class. The observation period used comprises 8.7 years of data between June 2008 and April 2017.

\section{Results of the MAGIC observations}

The MAGIC excess map above $200 \mathrm{GeV}$ reveals extended emission all over the remnant area as shown in figure 1. The emission forms an arc like structure in the north west with an adjunct bright extended source and additional hotspots inside the shell. The MAGIC emission largely overlaps with the extended emission seen by VERITAS. The strongest emission is located north of the $\mathrm{TeV}$ emission observed by VERITAS. Its centre lies on the radio limb, but extends beyond the shell. The arc like emission is aligned along the north-west part of the radio shell. The brightest emission spot inside the shell coincides with the brightest radio emission of the southern shell.

The MAGIC data can be well fitted with a flat disk model located at the centre of the radio shell $(\mathrm{Ra} / \mathrm{Dec}=(305.25 ; 40.43))$ with the same extension as the radio shell $\left(\mathrm{r}=0^{\circ} .56\right)$ and a $2 \mathrm{D}$ Gaussian centred on $(\mathrm{Ra} / \mathrm{Dec})=\left(304^{\circ} .95 \pm 0^{\circ} .01_{\text {stat }} \pm 0^{\circ} .02_{\text {sys }} ; 40^{\circ} .90 \pm, 0^{\circ} .02_{\text {stat }} \pm 0^{\circ} .02_{\text {sys }}\right)$ with an extension of $0^{\circ} .09 \pm 0.02$ (68\% containment radius) accounting for the bright source at the northern shell. The combined likelihood fit results in a significant $(>5 \sigma)$ detection for each source, the disk and the Gaussian. All remaining residuals are below $3 \sigma$ local significance. The extracted spectrum of the $\gamma$-Cygni SNR is consistent with previous measurement by Fermi-LAT and VERITAS [8].

The source shows an energy dependent morphology as displayed in figure 2. The emission below $200 \mathrm{GeV}$ is well confined within the supernova radio shell. However, the comparison with the $>200 \mathrm{GeV}$ measurements performed by MAGIC reveals a continuous change in the morphology: Above $450 \mathrm{GeV}$ the emission clearly extends beyond the radio shell by $\approx 0^{\circ} .2(6 \mathrm{pc}$ at $1.7 \mathrm{kpc})$. This behaviour is observed in the northern part of the shell only; however, the MAGIC observations were focussed on the northern part of the SNR and thus the exposure for the southern part is significantly lower. In addition at the southern part of the shell the sensitivity of MAGIC at 


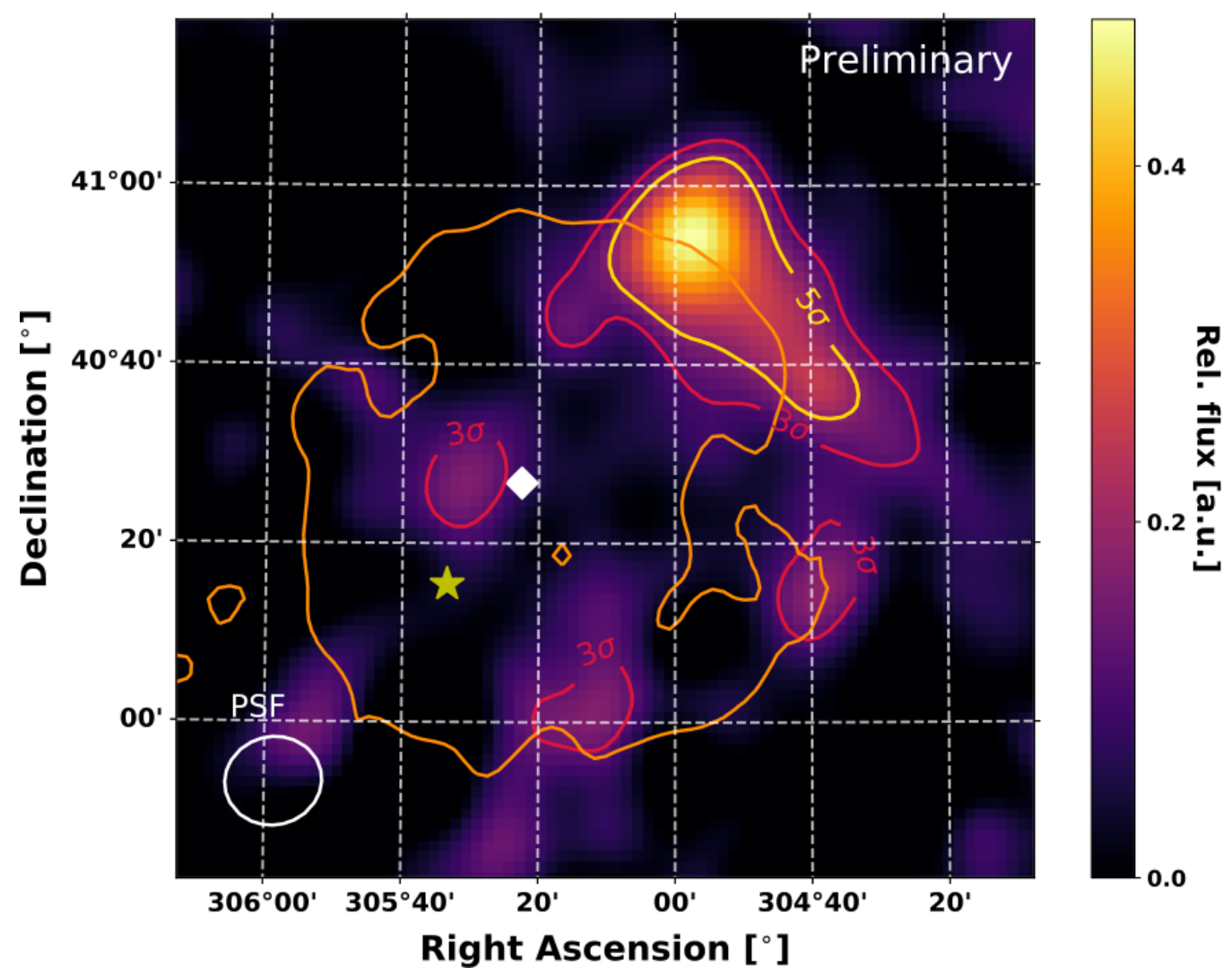

Figure 1: Relative flux map measured by MAGIC above $200 \mathrm{GeV}$ with 3 and $5 \sigma$ significance contours. The $400 \mathrm{~K}$ radio contours of the SNR from the $408 \mathrm{MHz}$ observation of the Canadian Galactic Plane Survey are added in orange. The white diamond indicates the position of PSR J2021+4026 and the yellow star the one of the $\gamma$-Cygni star, $\operatorname{Sadr}(2.2 \mathrm{mag})$.

lower energies is further reduced by the presence of Sadr. Therefore, a firm conclusion about the morphology of the southern shell cannot be derived yet.

\section{Discussion}

The complex morphology of the $\gamma$-Cygni region observed by MAGIC reveals intriguing details of the structure of this SNR. The brightest $\gamma$-ray emission originates from the north-west part of the shell, where X-ray measurements show bright thermal emission from shock heated gas [7] and hard X-ray clumps [15], of which at least one can be associated with the SNR and originating from non-thermal bremsstrahlung emission. Hence the emission hints at a denser interstellar medium towards the northern shell that is partially already overtaken by the shock. This may explain the concentration of the emission in the north-west in the GeV range as observed by Fermi-LAT. This speaks in favour of the hypothesis that the SNR expands inside the progenitor star bubble and starts to interact with a putative cavity wall as proposed by [16].

Additionally our observations show that the emission $>450 \mathrm{GeV}$ extends beyond the radio SNR shell by $\approx 0.2 \mathrm{deg}$. A possible explanation to this phenomenon are cosmic rays starting to escape the shock above several TeVs. This effect is widely discussed in the literature and expected 

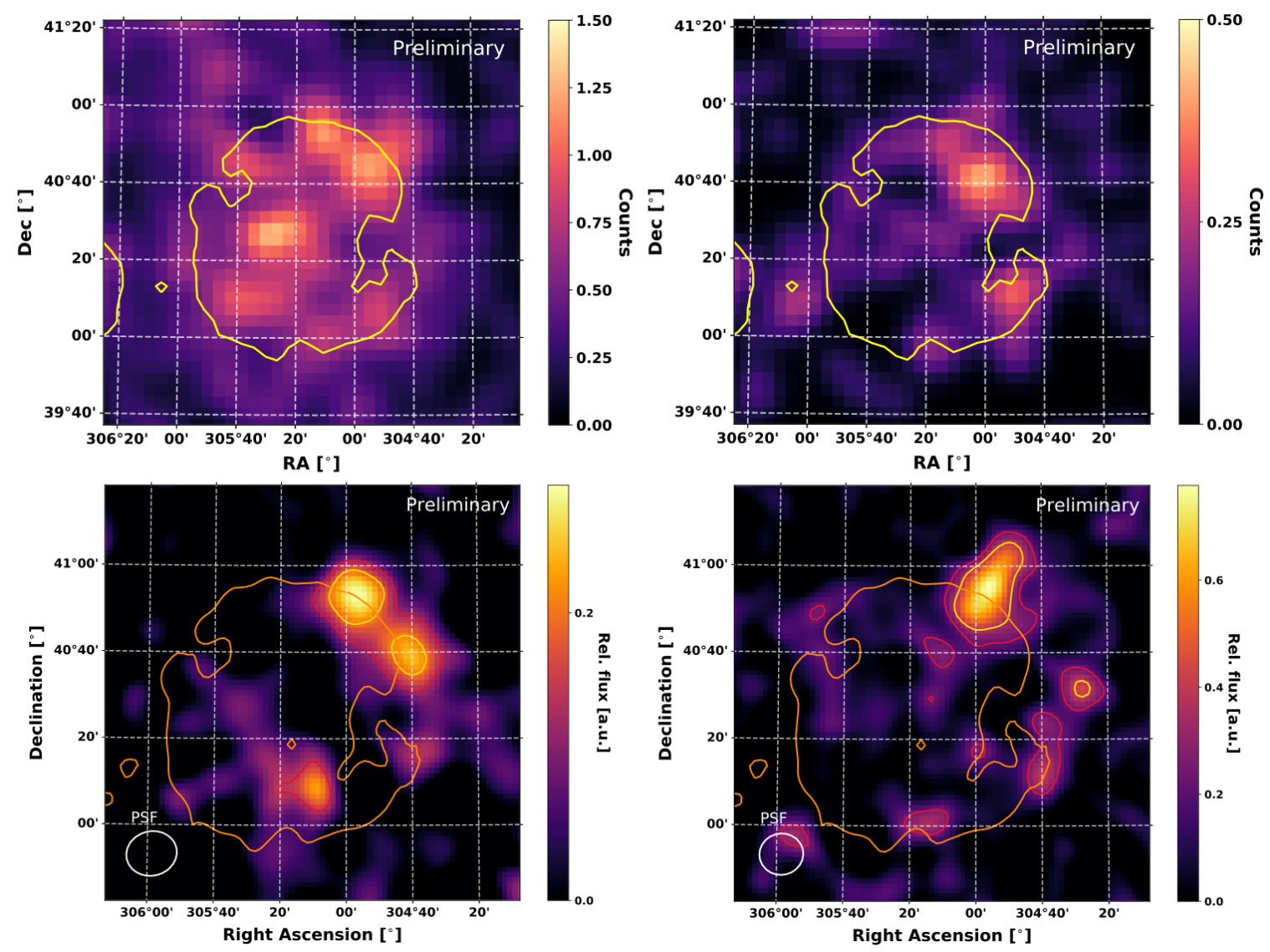

Figure 2: Morphology of the $\gamma$-ray emission at different energy bands. Upper left: photon count map of the $\gamma$-Cygni SNR between 15 and $50 \mathrm{GeV}$ as measured by Fermi-LAT smoothed with a Gaussian kernel of $\sigma=0^{\circ} .08$. The yellow line is the $400 \mathrm{~K}$ contour of the CGPS radio measurement at $408 \mathrm{MHz}$. Upper right: same as left image, but in an energy range from 50 to $200 \mathrm{GeV}$. Lower left: relative flux map as measured by MAGIC in the energy range from 200 to $450 \mathrm{GeV}$ and the orange line is the same radio contour as in the upper panels. Lower right: same as the image to the left, but at energies above $450 \mathrm{GeV}$.

for a Sedov phase SNR (e.g. [17]). The transition energy suggested by the MAGIC observations lies in the expected energy range for $\mathrm{a} \approx 7000 \mathrm{yrs}$ old SNR. Either the cavity wall or a CO cloud visible in the $\mathrm{J}=2 \rightarrow 1$ line emission towards the main emission region seen by MAGIC observed by the Planck satellite ${ }^{1}$ could be a target for escaping cosmic rays.

Despite this plausible explanation, the association of the observed $\gamma$-ray emission with the SNR can be questioned. Indeed, while the association of the larger scale shell emission with $\gamma$ Cygni seems to be justified as it is aligned along the radio shell, the bright source in the north-west can be of different origin. Still there are no known AGNs or PWN within 0.2 deg from the position of the source requiring it to be an "dark accelerator". Also there is no variability observed from this object. Hence, the association with the $\gamma$-Cygni SNR currently seems the most likely one.

\footnotetext{
${ }^{1}$ http://pla.esac.esa.int/pla/
} 


\section{Conclusion}

MAGIC observations have shown that the $\gamma$-ray emission from $\gamma$-Cygni shows a complex, energy dependent morphology. They suggest that the SNR consists of different components dominating the emission at different energy bands. At $\lesssim 450 \mathrm{GeV}$ the emission seems to be confined inside the radio shell agreeing with the emission observed by Fermi-LAT. Towards higher energies the emission seems to be concentrated at the rim of the northern shell and extending beyond it. This two component model can explain the difference in the spectra measured by Fermi-LAT and VERITAS and is hinting at an enhanced interaction of the SNR shock with the interstellar medium at the northern shell and a possible leakage of cosmic rays into the ambient medium.

\section{Acknowledgments}

We would like to thank the IAC for the excellent working conditions at the ORM in La Palma. We acknowledge the financial support of the German BMBF, DFG and MPG, the Italian INFN and INAF, the Swiss National Fund SNF, the European ERDF, the Spanish MINECO, the Japanese JSPS and MEXT, the Croatian CSF, and the Polish NCN.

\section{References}

[1] M. Ackermann, M. Ajello, A. Allafort, L. Baldini, J. Ballet, G. Barbiellini et al., Detection of the Characteristic Pion-Decay Signature in Supernova Remnants, Science 339 (Feb., 2013) 807-811.

[2] S. Funk, Ground-and Space-Based Gamma-Ray Astronomy, Annual Review of Nuclear and Particle Science 65 (Oct., 2015) 245-277.

[3] C. Y. Hui, K. A. Seo, L. C. C. Lin, R. H. H. Huang, C. P. Hu, J. H. K. Wu et al., A Detailed X-Ray Investigation of PSR J2021+4026 and the $\gamma$-Cygni Supernova Remnant, The Astrophysical Journal 799 (Jan., 2015) 76.

[4] A. Allafort, L. Baldini, J. Ballet, G. Barbiellini, M. G. Baring, D. Bastieri et al., PSR J2021+4026 in the Gamma Cygni Region: The First Variable gamma-Ray Pulsar Seen by the Fermi LAT, The Astrophysical Journal Letters 777 (Nov., 2013) L2.

[5] D. A. Green, A catalogue of 294 Galactic supernova remnants, Bulletin of the Astronomical Society of India 42 (June, 2014) 47-58.

[6] T. A. Lozinskaya, V. V. Pravdikova and A. V. Finoguenov, The Supernova Remnant G78.2+2.1: New Optical and X-ray Observations, Astronomy Letters 26 (Feb., 2000) 77-87.

[7] D. A. Leahy, K. Green and S. Ranasinghe, X-ray and radio observations of the gamma Cygni supernova remnant G78.2+2.1, Monthly Notices of the Royal Astronomical Society 436 (Dec., 2013) 968-977.

[8] E. Aliu, S. Archambault, T. Arlen, T. Aune, M. Beilicke, W. Benbow et al., Discovery of TeV Gamma-Ray Emission toward Supernova Remnant SNR G78.2+2.1, The Astrophysical Journal 770 (June, 2013) 93.

[9] J. Lande, M. Ackermann, A. Allafort, J. Ballet, K. Bechtol, T. H. Burnett et al., Search for Spatially Extended Fermi Large Area Telescope Sources Using Two Years of Data, The Astrophysical Journal 756 (Sept., 2012) 5. 
[10] N. Fraija and M. Araya, The Gigaelectronvolt Counterpart of VER J2019+407 in the Northern Shell of the Supernova Remnant G78.2+2.1 ( $\gamma$ Cygni), The Astrophysical Journal 826 (July, 2016) 31.

[11] J. Aleksić, S. Ansoldi, L. A. Antonelli, P. Antoranz, A. Babic, P. Bangale et al., The major upgrade of the MAGIC telescopes, Part II: A performance study using observations of the Crab Nebula, Astroparticle Physics 72 (Jan., 2016) 76-94.

[12] C. Fruck, M. Gaug, R. Zanin, D. Dorner, D. Garrido, R. Mirzoyan et al., MARS, the MAGIC analysis and reconstruction software, ArXiv e-prints 1403 (Mar., 2014) arXiv:1403.3591.

[13] J. Albert, E. Aliu, H. Anderhub, P. Antoranz, A. Armada, M. Asensio et al., Implementation of the Random Forest method for the Imaging Atmospheric Cherenkov Telescope MAGIC, Nuclear Instruments and Methods in Physics Research A 588 (Apr., 2008) 424-432.

[14] J. R. Mattox, D. L. Bertsch, J. Chiang, B. L. Dingus, S. W. Digel, J. A. Esposito et al., The Likelihood Analysis of EGRET Data, The Astrophysical Journal 461 (Apr., 1996) 396.

[15] Y. Uchiyama, T. Takahashi, F. A. Aharonian and J. R. Mattox, ASCA View of the Supernova Remnant gamma Cygni (G78.2+2.1): Bremsstrahlung X-Ray Spectrum from Loss-flattened Electron Distribution, The Astrophysical Journal 571 (June, 2002) 866-875.

[16] Y. Ladouceur and S. Pineault, New perspectives on the supernova remnant G78.2+2.1, Astronomy and Astrophysics 490 (Oct., 2008) 197-211.

[17] D. Caprioli, P. Blasi and E. Amato, On the escape of particles from cosmic ray modified shocks, Monthly Notices of the Royal Astronomical Society 396 (July, 2009) 2065-2073. 